\title{
Ambient Sensorization for the Furtherance of Sustainability
}

\author{
Fábio Silva, Cesar Analide, Luís Rosa, Gilberto Felgueiras, Cedric Pimenta \\ fabiosilva, analide\{ddi.uminho.pt\}, luisrosalerta,gil.m.fell, \\ cedricpim \{lgmail.com\}
}

\begin{abstract}
Energy efficiency is regarded as an important objective in a world of limited resources. The sustainable use of energy is necessary for the continuity of life styles that do not jeopardize the future. Nevertheless, due to poor information about the impact of human actions on the environment, it is hard to promote and warn for sustainability. This work focuses on the use of ambient intelligence as a mean to constantly revise sustainability indicators in a way they may be used for user awareness and recommendation systems within communities. The approach in this research makes use of sustainable indicators monitored through ambient sensors which enable user accountability concerning their actions inside each environment. Also, it is possible to compare the effect of user actions in the environment, enabling decision making based on such comparison factors.
\end{abstract}

Keywords. Ambient Intelligence, Sustainability, Energy Efficiency, User Awareness

\section{Introduction}

Energy efficiency represents optimal use of energy to satisfy the objectives and needs from users, environments and interactions between them. According to Herring studies [1], over the last 25 years, the increase in the efficiency of domestic appliances has been nullified by the increase of the use of energy consumption devices. Initial results from energy efficiency policies state that small changes in habits can save up to $10 \%$ in home energy consumption [2]. On the other hand, sustainability represents the assurance that environments, users and interaction between them can be endured and, as a consequence, the future replication of the current patterns is not compromised.

Both concepts, sustainability and energy efficiency, are not opposed to the use of energy, but they do remind people to be effective on how resources are used and the fact that sustainability concerns the viability of current actions in the present and in the future. Currently, different approaches to measure and assess sustainability are addressed in the literature. Some focus on an economic perspective while others emphasize environmental or social perspectives [3]. On a computer science perspective, although not being able to directly solve the sustainability problem, it can plan and develop solutions to measure and assess sustainability automatically from an envi- 
ronment. This is not due without obtaining information about the environment and its users. The scientific research field of Ambient Intelligence provides a wide spectrum of methodologies to obtain such information in a non-intrusive manner.

The types of sensors used in the environment may be divided into categories to better explain their purpose. Generally, an ambient might be divided by sensors and actuators. Sensors monitor the environment and gather data useful for cognitive and reasoning processes [4]. Actuators take action upon the environment, performing actions such as controlling the temperature, the lightning or other appliances. In terms of sensorization, environment sensors can be divided into sensor that monitor environment or sensor that monitor the user and its activities.

This division of sensor classes can also be presented in a different form, taking into consideration the role of the sensor in the environment [5]. In this aspect, sensors might be divided into embedded sensors are installed on objects, context sensors provide information about the environment, or motion sensors.

The work here presented considers the use of these three types of sensors to assess and reason about sustainability and energy efficiency.

The use of indicators for sustainability assessment is a common practice across many researchers. Nevertheless, the definition of a sustainable indicator is sometimes difficult and it may differ from environment to environment. In intelligent buildings, there are proposals to build Key Performance Indicators (KPIs) to monitor sustainability and act as sustainable indicators [6]. It has also been identified that indicators are useful at pointing unsustainable practices but not so accurate nor useful to define and guarantee sustainability [7]. Frameworks to evaluate energy efficiency through sustainability in the literature use similar approaches. The goal of energy efficiency was obtained optimizing sustainable indicators which monitor a set of specific energy sources [8]. Industrial environments are also object of energy efficiency projects. In Heilala et al. [11], an industrial AmI is proposed to optimize energy consumption. The main technique used by the AmI system is based on case based reasoning, comparing the data gathered and processed in the AmI with EUP values to assess and diagnose possible inappropriate energy usages. An intelligent decision support model for the identification of intervention needs and further evaluation of energy saving measures in a building is proposed Doukas et al. [7]. The demonstrated concept shows that it is possible to have an intelligent model to perform energy management on a building, combining aspects like ambient climate conditions, investment rates, fuel, and carbon prices, and, also, past experiences.

\section{Sustainability}

\subsection{Definition}

Sustainability is a multidisciplinary concept related with the ability to maintain support and endure something at a certain rate or level. The United Nations have defined this concept as meeting the needs of the present without compromising future generation to meet their own needs. Due to the importance of sustainability, different authors have defined measures to assess and characterize sustainability. A popular consensus 
is based on 3 different indicators, used to measure the sustainability of a given environment [9]. This approach is based on three different types of indicators, social, economic and environmental, with the specific restriction that until all those values are met, a system cannot be deemed sustainable. From this perspective, sustainability concerns a delicate equilibrium between different indicators, where actions to optimize one indicator might affect anyone of the other two. As a consequence, sustainability planning becomes a hard problem, involving multi-objective optimization techniques, whereas the best solution might not concern the optimization of individual indicators, but rather a compromise between all of them.

\subsection{Human response to sustainability}

It is intended to have users involved and motivated to the sustainability issues, even when some distress may arise from its experience on the environment. Thus, to improve the user's approach to sustainability management platform, it is necessary to reduce this emotional distress. To do so it is necessary to replicate human behavior and emotions, approaching psychological models.

Affective Computing is a computational area that provides techniques for the simulation of emotions, personalities and behaviors, introduced by Piccard. This simulation concludes that cognitive and affective states of humans can reduce the nondeterminism of decision making of robots and virtual characters by giving them another level of intelligence [10]. To simulate emotions, models such as the OCC model [11] and the PAD space [12] are common among computational researchers. For personalities, the big five factor model explains how personality is constructed mapping values into five variables: extraversion, agreeableness, conscientiousness, openness, neuroticism, being possible to replicate an human personality [13].

Emotion and personality are intrinsic characteristics of the behavior control, so it is necessary to process the information of an external event. Kazemifard et al. present a model to do that [14]. They separate the information processing into three levels such as reactive level that receives external information triggering a proto-emotion like the associative component of thinking, a reflective level that receives internal information like the unconscious and a routine level, a rule-based component which controls the other levels. This computational model interprets the flow of information from external events to internal change of cognitive and emotional states which might open the possibility to correspond the changes of sustainability indicators to user behavior.

\subsection{Sustainable Indicators}

The sustainability of a system may be pointed out by a set of indicators, as suggested by many authors, allowing the definition and monitoring of indicators. However, there are common problems with this practice, enumerated in the literature, [8]. The definition of global sustainable indicators, as a means to compare environments, is difficult since environments have different characteristics. Selection and formal definition of indicators is, also, a matter of concern as it has to be agreed by all intervenients and must have a series of properties, in which the indicators express their rele- 
vance. Some authors approach this problem characterizing these properties as dimensions, where some indicators are more important in some dimensions than in others, while monitoring the same object. One other problem is the definition of measuring units and metadata. If not defined accordingly, it may be impossible to compare indicators of the same type. Measuring data makes it possible to obtain an indicator which might have a range of optimal values and a range of non-optimal values.

Finally, the presence of indicators to assess sustainability is a common practice. However it does not give any information on how to guarantee or plan sustainability. In fact, indicators only inform about the current status of a system. This work focuses on using sustainable indicators that are built using a common strategy with the same units within the same range of values to facilitate integration with learning and ranking algorithms from ambient intelligence.

\section{Sustainability Assessment with Ambient Intelligence}

\subsection{Sustainability Assessment}

As detailed in section 2, indicators are able to detect inefficiencies, but they cannot provide means to guarantee sustainable actions. Therefore, their construction should facilitate their integration on reasoning platforms and algorithms, so they can be used to help achieving sustainability and improving solutions [15]. In this work, the assessment of sustainability focuses on three key dimensions: economic, environmental and social. Within each category, indicators are defined to monitor interest variables inside each category. Indicators are built measuring the positive and negative impact of key variables in the system, and their values are defined as shown in equation (1).

$$
\text { Indicator(positive, negative })=\left\{\begin{array}{l}
\frac{\text { positive }}{\text { negative }}-1 \rightarrow \text { positive }<=\text { negative } \\
1-\frac{\text { negative }}{\text { positive }} \rightarrow \text { positive }>\text { negative }
\end{array}\right.
$$

In this proposal, each indicator as a common scale representing a ratio defined in the interval $[-1 ; 1]$, where negative values represent unsustainability and positive values respect to sustainability. Moreover, it is possible to aggregate values using simple averaging functions, from a small to a larger perspective. All these indicators are calculated either locally, i.e., in a room basis, or globally, i.e., environment. Thus, even if the environment is considered sustainable, the user may still assess changes in premises with unsustainable values.

Table 1. Sustainability Indicators

\begin{tabular}{|c|c|c|c|c|}
\hline & & Economic & Environmental & Social \\
\hline \multirow{2}{*}{$\begin{array}{c}\text { Sample } \\
\text { Indicator }\end{array}$} & Positive & Budget & Emissions Avoided & Time Inside \\
\hline & Negative & Cost & Emissions & Time Outside \\
\hline
\end{tabular}


The formal definition of indicators is an active research field. There are different proposals for indicator selection and definition [6], [8]. With the purpose of testing the definition of indicators with the strategy presented in section 2.1 sample indicators for each dimension of sustainability were created as demonstrated in table 1 . These sample indicators were used to perform experiments in section 4. In order to deliberate about sustainability performance it is needed to rank solutions, by rewarding each solution with a sustainable score. This score can then be used to assess and compare environments inside communities, helping users improve their scores by sharing good behaviors across social network platforms, which presents the users with examples of the best scoring solutions, so they can improve their score.

$$
\begin{gathered}
\mathrm{S}_{\text {index }}=\alpha * \mathrm{I}_{\text {economic }}+\beta * \mathrm{I}_{\text {enverinmental }}+\gamma * \mathrm{I}_{\text {social }} \\
\alpha+\beta+\gamma=1 \wedge 0<\alpha<1 \wedge 0<\beta<1 \wedge 0<\gamma<1
\end{gathered}
$$

The ranking system was designed to take into account the three dimensions of sustainability averaging them with variable weights per dimension as expressed by equation 2. Although the dimension weights should ideally be equal, the expression designed allows the discrimination to account for the promotion of strategies.

\subsection{People Help Energy Savings and Sustainability (PHESS)}

PHESS is a research project under development at University of Minho which aims to measure the sustainability of environments and its users. The approach focuses on the user and its interactions with environments, assessing their impact in terms of sustainability. The main objective is to build an ambient intelligence platform to promote overall energy efficiency and sustainability.

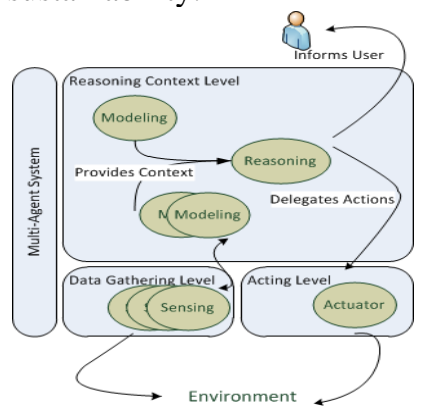

Fig. 1. Multi-Agent System for Deliberation and Sustainable Assurance

Thus, the sustainability assessment used in this work was embedded in a multi-agent system (figure 1) that has, as its primary mission, the management of data and information flow across the community of users, and the promotion of sustainable behaviors. PHESS is built upon a multi-agent system divided into 3 components: data gathering, reasoning and actuating. The data gathering component is composed of agents, sensing agents, responsible for constantly monitoring the environment. All data transformation is made in the reasoning component, as well as the indicators definition and 
calculation, per environment and user. In this component there are two types of agents: a model agent that models data coming from sensing agents which combines data from sensors to environment and user representations in the agents of this layer. Reasoning agents use agents that model users and environments to recreate simulations combining both where it is possible. It extracts information from both models and combines those models in the simulation engine to test sustainability hypothesis through the values of sustainability indicators. The acting level uses information processed by the reasoning context level to propose and alert users.

\section{$4 \quad$ Results}

A number of validations using the platform were designed and implemented in order to prove the practicability and usability of the concepts detailed. As a result, the platform was integrated in simulated, controlled and restricted environments. It was responsible for sensorization and reasoning generating reports and recommendations to users with the aim of reaching better levels of sustainability and energy efficiency.

Table 2. Simulation results for user and environent tracking

\begin{tabular}{|r|c|c|c|}
\hline \multicolumn{5}{|l|}{ Environment 1 } & Social & Economic & Environmental \\
\hline Kitchen & -0.9011 & -0.6859 & -0.3263 \\
\hline Bedroom & 0.1818 & 0.9936 & -0.3263 \\
\hline Living Room & -0.5294 & 0.1040 & -0.3263 \\
\hline Hall & -0.9690 & 0.9968 & -0.3263 \\
\hline WC & -0.9900 & 0.9968 & -0.3263 \\
\hline Environment 2 & Social & Economic & Environmental \\
\hline & -0.8889 & -0.6231 & -0.3263 \\
\hline Kitchen & 0.0833 & 0.9946 & -0.3263 \\
\hline Bedroom & -0.4849 & 0.2533 & -0.3263 \\
\hline Living Room & -0.9690 & 0.9968 & -0.3263 \\
\hline Hall & -0.9900 & 0.9968 & -0.3263 \\
\hline WC & \multicolumn{1}{|l}{}
\end{tabular}

For testing purposes, full-fledge environments were defined with a set of rooms commonly found under residential setups. The two environments simulated contained a total of 5 rooms, a bedroom, a living-room, a kitchen, a bathroom and a hall providing connection between all the other rooms. Appliances were defined ranging from lights and computers to ovens and refrigerators with different consumption models in each environment. Consumption of appliances was defined from their active use and explicit power on/off actions from simulated user actions and default consumption models. User actions included movement between rooms and appliance switching on and off. Although, the user performed the same generic action their duration, time and order was different, as well as, the time each user spends inside the environment. 
Table 2 demonstrates the user report generated by the PHESS system on two users under different environments for one full day.

In order to produce the report an average cost was defined for each room and presence indicators represent the total time with users inside the room versus total time without users in the room. This simulation demonstrates the identification through the use of sustainable indicators, the pattern of sustainability in two environments. Due to the fact that both environments where used by only one user, the social indicator has negative values for most of the room because they are mostly vacant, although the environment itself contains users on it. This results in the difference in the social indicator on a room by room analysis and environment analysis. Environments with more users would help the value of the social indicator if more rooms become occupied at the same time. Regarding the environmental indicator, it is calculated from electrical consumption. As both environments have the same electrical source, they have the same value for this indicator. This indicator may vary if the electricity provider user more or less green sources. The economic indicator is directly connected with the price of electrical consumption and the amount available.

Table 3. Taking Advantage of Information and Knowledge

\begin{tabular}{|l|l|l|l|}
\hline Environment 1 & Social & Economic & Environmental \\
\hline User 1 & -0.004 & 0.3241 & -0.586 \\
\hline User 2 & 0.5 & 0.927 & -0.586 \\
\hline Environment 2 & Social & Economic & Environmental \\
\hline User 1 & -0.004 & 0.2016 & -0.586 \\
\hline User 2 & 0.5 & 0.827 & -0.586 \\
\hline
\end{tabular}

Using PHESS system and user models created it is also possible to recreate one user behavior in other environments, provided environments are compatible as it is the case. Re-running the simulation using stored user behaviors showcases the potential to compare the effect on specific user behaviors in different environments and how environment and sustainability indicators are affected. In table 3 , it is demonstrated that user 2 would perform better environment 1 while user 1 would not be as sustainable in environment 2 , thus making environment 2 considered less sustainable for that group of users. Moreover, information collected efficiently aggregates different indicators into each dimension of sustainability as defined in section 3 providing comprehensible information to the user.

\section{Conclusion}

The use of AmI technology is a valid effort to track sustainability on a real time basis, enabling user accountability for their action inside environments. The results provided in this work demonstrate that it is possible to use a sustainability assessment to directly compare the sustainability performance of both users and environments. Furthermore, the use of AmI techniques enables users and environments profiling testing if a better distribution of users per environments results in better sustainable indicators. 
In the future, there is the need to integrate more reasoning context with current and past indicators to create recommendations on the platform. Moreover, the definition of more sustainability indicators is necessary to test the robustness of the sustainability assessment engine. Also, the deployment of the PHESS system is scheduled in order to test the findings found with real environments and users.

Acknowledgements. This work is funded by National Funds through the FCT Fundação para a Ciência e a Tecnologia (Portuguese Foundation for Science and Technology) within projects PEst-OE/EEI/UI0752/2011 and PTDC/EEISII/1386/2012. It is also supported by a doctoral grant, SFRH/BD/78713/2011, issued by the Fundação da Ciência e Tecnologia (FCT) in Portugal.

\section{References}

1. H. Herring, "Energy efficiency-a critical view," Energy, vol. 31, no. 1, pp. 10-20, 2006.

2. M. Chetty and D. Tran, "Getting to Green : Understanding Resource Consumption in the Home," in Proceedings of the 10th international conference on Ubiquitous computing, 2008, pp. 242-251.

3. R. Singh, H. Murty, S. Gupta, and A. Dikshit, "An overview of sustainability assessment methodologies," Ecological Indicators, vol. 9, no. 2, pp. 189-212, 2009.

4. A. Aztiria, A. Izaguirre, and J. C. Augusto, "Learning patterns in ambient intelligence environments: a survey," Artif. Intell. Rev., vol. 34, no. 1, pp. 35-51, May 2010.

5. A. Aztiria, J. C. Augusto, R. Basagoiti, A. Izaguirre, and D. J. Cook, "Discovering frequent user-environment interactions in intelligent environments," Personal and Ubiquitous Computing, vol. 16, no. 1, pp. 91-103, 2012.

6. H. Al-Waer and D. J. Clements-Croome, "Key performance indicators (KPIs) and priority setting in using the multi-attribute approach for assessing sustainable intelligent buildings," Building and Environment, vol. 45, no. 4, pp. 799-807, 2009.

7. A. Lyon and Dahl, "Achievements and gaps in indicators for sustainability," Ecological Indicators, vol. 17, no. 0, pp. 14-19, 2012.

8. N. H. Afgan, M. G. Carvalho, and N. V Hovanov, "Energy system assessment with sustainability indicators," Energy Policy, vol. 28, no. 9, pp. 603-612, 2000.

9. V. Todorov and D. Marinova, "Modelling sustainability," Mathematics and Computers in Simulation, vol. 81, no. 7, pp. 1397-1408, 2011.

10. R. W. Picard, Affective Computing. Cambridge: The MIT Press, 1997.

11. A. Ortony, G. Clore, and A. Collins, The cognitive structure of emotions. 1990.

12. P. Gebhard, "ALMA: a layered model of affect," Proceedings of the fourth international joint conference on Autonomous agents and multiagent systems, pp. 29-36, 2005.

13. R. R. McCrae and O. P. John, "An introduction to the five-factor model and its applications.," Journal of personality, vol. 60, no. 2, pp. 175-215, Jun. 1992.

14. M. Kazemifard, N. Ghasem-Aghaee, and T. I. Ören, "Emotive and cognitive simulations by agents: Roles of three levels of information processing," Cognitive Systems Research, vol. 13, no. 1, pp. 24-38, Mar. 2012.

15. F. Silva, D. Cuevas, C. Analide, J. Neves, and J. Marques, "Sensorization and Intelligent Systems in Energetic Sustainable Environments," in Intelligent Distributed Computing VI, 2013, vol. 446, pp. 199-204. 